\title{
ANÁLISE DO NÍVEL DE SUSTENTABILIDADE DAS UNIDADES FEDERATIVAS DO BRASIL E DE SUAS CAPITAIS: UM ESTUDO SOB AS PERSPECTIVAS ECONÔMICA, SOCIAL E AMBIENTAL
}

\author{
Marcelo Alvaro da Silva Macedo \\ Doutor em Engenharia de Produção Universidade Federal do Rio de Janeiro - COPPE/UFRJ \\ Professor da Universidade Federal do Rio de Janeiro - UFRJ \\ malvaro.facc.ufrj@gmail.com
}

Antonio Francisco Ritter Ferreira

Mestre em Gestão e Estratégia em Negócios Universidade Federal do Rio - MPGE/UFRRJ

Professor do Departamento de Ciências Administrativas e do Ambiente - UFRRJ

afritter@yahoo.com.br

Fabricio Carvalho Cípola

Mestre em Gestão e Estratégia em Negócios Universidade Federal do Rio de Janeiro - UFRRJ Professor da Universidade Federal do Rio de Janeiro - UFRJ

fcipola@terra.com.br

\section{RESUMO}

Quando uma região consegue resolver de forma integrada questões sobre crescimento econômico, exploração de recursos naturais, pobreza e distribuição de renda, que têm natureza econômica, ambiental e social, ela se torna, então, sustentável, pois se mostra capaz de promover, de forma equilibrada e harmônica, crescimento econômico, qualidade de vida e respeito ao meio ambiente. Nesse contexto, o presente artigo analisa o nível de sustentabilidade das 27 unidades federativas (UFs) do Brasil e de suas capitais. Para tanto, foi aplicada a metodologia de Análise Envoltória de Dados (DEA) a informações de natureza econômica, social e ambiental, que são os parâmetros do TBL - Triple Bottom Line, no intuito de gerar um indicador de sustentabilidade denominado Índice Consolidado de Desenvolvimento Sustentável (ICDS). Os resultados mostram que o Distrito Federal e a cidade de Vitória no Espírito Santo são destaques positivos, enquanto que o Maranhão e a cidade de Maceió em Alagoas obtêm resultados ruins em termos de desenvolvimento sustentável. Uma questão relevante, que surge da comparação destes rankings é a posição dos estados de Alagoas e do Piauí, que possuem enquanto UFs desempenhos ruins e que têm suas capitais também com baixo nível de sustentabilidade. Esta é realmente uma situação preocupante e que merece uma intervenção urgente, com o desenvolvimento de políticas públicas que envolva as instâncias municipal, estadual e federal.

Palavras-chave: Desenvolvimento Sustentável; Sustentabilidade; TBL; DEA.

\section{ANALYSIS OF THE LEVEL OF SUSTAINABILITY FEDERATION UNITS BRAZIL AND ITS CAPITAL: A STUDY IN PERSPECTIVE ECONOMIC, SOCIAL AND ENVIRONMENTAL}

\section{ABSTRACT}

When a region can resolve integrated issues about economic growth, resource exploitation, poverty and income distribution, that have economic, environmental and social perspectives, it becomes, then, sustainable, since it is able to promote balanced and harmonious economic growth, quality of life and respect for the environment. In this context, this paper analyzes the sustainability level of 27 federal units (UFs) of Brazil and theirs capitals. For this, we applied the Data Envelopment Analysis (DEA) methodology to information about economic, social and environmental issues, that are the TBL parameters - Triple Bottom Line, in order to generate an indicator of sustainability called Sustainable Development Consolidated Index (ICDS). The results show that the Distrito Federal and the city of Vitoria in Espirito Santo are top gainers, while the Maranhão and the city of Maceió in Alagoas get bad results in terms of sustainable development. A relevant question, that emerges from the comparison of these rankings, is the position of the states of Alagoas and Piaui. They have bad performance while UFs and theirs capitals also have low level of sustainability. This is really a worrying situation and one that deserves urgent intervention, with the development of public policies involving the municipal, state and federal authorities.

Keywords: Sustainable Development; Sustainability; TBL; DEA.

\section{INTRODUÇÃO}


Segundo Souza, Andrade e Cândido (2008) no final do século XX, verificou-se um alerta quanto à capacidade da natureza continuar oferecendo todos os recursos necessários para a sobrevivência da humanidade. Por um lado, o crescimento da população, por si só, já permite que muitos dos recursos naturais se esgotem em poucos anos. De forma paralela, o consumo desordenado e a exploração das riquezas naturais sem a devida reposição têm contribuído para o estágio atual de degradação sem precedente na história, sendo decorrente do processo de desenvolvimento econômico corrente.

Neste contexto, surge a discussão de desenvolvimento sustentável, que segundo a WCED (1987), é aquele que atende às necessidades das gerações atuais sem comprometer a capacidade de atendimento das necessidades das gerações futuras. Este deve estar inserido, de acordo com Costanza (1991), num contexto de relação entre as dinâmicas econômica, social e ambiental, onde tem-se a constante busca pela eficiência econômica, a justiça social e a qualidade ambiental. Em outras palavras, Souza et al. (2008), dizem que não existe mais espaço para que o capital econômico consiga se acumular e reproduzir sustentavelmente onde não exista um conjunto de outras dimensões, baseadas, principalmente, em aspectos sociais e ambientais.

De acordo com Ruthes e Nascimento (2006), regiões sustentáveis são aquelas que conseguem promover, de forma equilibrada e harmônica, crescimento econômico, qualidade de vida e respeito ao meio ambiente. A idéia é promover progresso econômico de forma responsável, tanto socialmente quanto ambientalmente. Assim sendo, como destaca Jacobi (1994), a busca pela sustentabilidade envolve, simultaneamente, resolver de forma interrelacionada questões complexas, tais como crescimento econômico, exploração de recursos naturais, pobreza e distribuição de renda, que têm natureza econômica, ambiental e social.

Lima, Gómez e Catillo (2008) ressaltam a abrangência destas três perspectivas: econômica, social e ambiental, na formação do que Sachs (2007) denominou de ecossocioeconomia. A perspectiva econômica trata da alocação e distribuição eficiente dos recursos naturais em uma escala apropriada. Já a perspectiva social, refere-se à preocupação com o bem-estar humano, através do acesso a serviços básicos: água limpa e tratada, ar puro, serviços médicos, segurança, proteção e educação, dentre outros. Por fim, a perspectiva ambiental representa a utilização do potencial encontrado nos diversos ecossistemas, com um nível mínimo de deteriorização dos mesmos, de forma a não comprometer sua existência a longo prazo.

Neste sentido, o problema de pesquisa se constitui em como analisar o nível de sustentabilidade de cada unidade federativa (UF) do Brasil e de suas capitais frente a múltiplas perspectivas/dimensões da sustentabilidade.

Sendo assim, o objetivo do artigo é verificar o nível de sustentabilidade de cada UF do Brasil e de suas capitais, de forma comparativa e multicriterial, com a aplicação da Análise Envoltória de Dados (DEA), levando em conta vetores de sustentabilidade de naturezas distintas, no caso econômico, social e ambiental, que formam as perspectivas do Triple Bottom Line (TBL). Neste sentido, a questão de pesquisa é: Qual o nível de sustentabilidade de cada UF e de cada Capital, considerando as perspectivas econômica, social e ambiental combinadas?

\section{DESENVOLVIMENTO SUSTENTÁVEL}

Para Aligleri, Aligleri e Krugliankas (2009), apesar dos já vinte anos da Constituição Cidadã, que entre tantas inovações consagra o direito a que todos tem ao meio ambiente ecologicamente equilibrado, bem de uso comum do povo e essencial à sadia qualidade de vida, confirmando e fortalecendo a idéia de que o interesse na defesa e preservação do ambiente é dever do Poder Público e da coletividade, incluída a iniciativa privada, temas como gestão ambiental, gestão socioambiental, sustentabilidade empresarial ainda são considerados novos no dia a dia dos gestores e principalmente nos trabalhos da Academia.

Macedo (2009) afirma que a sustentabilidade ganhou notoriedade e passou a fazer parte da agenda global de preocupações, a partir da constatação, na passagem do século XX para o século 
XXI, de que os padrões de produção e consumo não poderiam permanecer nos patamares elevados em que se encontravam, considerando os recursos à disposição. A realidade de então mostrava que a manutenção dos patamares de consumo, exploração dos recursos naturais e uso de energia geraria degradações socioambientais de altas proporções e irreversíveis, gerando incertezas até em relação ao futuro da humanidade.

Nascimento (2008) ilustra que nas últimas décadas as previsões sobre o futuro da humanidade foram baseadas em dados científicos e, apesar das polêmicas, sensibilizaram grande parte da população mundial quanto à gravidade de problemas como o aquecimento global. Neste sentido, com a disseminação dos meios de comunicação e da Internet, o assunto foi ganhando espaço no dia a dia das pessoas.

O crescimento da população e a elevação do consumo aumentam a demanda de recursos naturais finitos, extremamente poluentes, como o carvão, o gás e o petróleo. Além disso, para Grayson e Hodges (2002) a seca em grandes regiões do planeta aumenta a procura por água, que começa a se tornar escassa face o aumento da poluição dos rios, mares e lagoas.

Rodrigues, Santos e Mello (2009) salientam que os recursos naturais, considerados superabundantes outrora, começam a dar sinais de esgotamento, com profundas transformações no meio ambiente natural, em que a civilização humana se desenvolveu. Segundo os autores, os anos foram passando e os especialistas perceberam que os recursos naturais podem se extinguir devido ao uso descontrolado e o imediatismo dos resultados. Contudo, a utilização irresponsável dos meios naturais para a produção de bens e serviço tende a reverter negativamente para as entidades, pois hoje a sociedade repudia quem provoca danos ambientais.

Nas palavras do WCED (1987), a preocupação com o futuro do planeta, com as pessoas e o meio ambiente já se mostrava latente. Problemas como pobreza, crescimento rápido e desregulamentado das corporações e mercados, sobrevivência, enfraquecimento do Estado e crises econômicas geravam um ambiente de grande incerteza quanto ao futuro do planeta e as das gerações vindouras.

Para Lima (2005), sem renda não há consumo, sem educação não há mão-de-obra qualificada, sem saúde não há trabalhador produtivo e sem emprego, não há desenvolvimento. Diante deste cenário, importantes organismos internacionais como o BIRD - Banco Mundial, o BID - Banco Interamericano de Desenvolvimento, o FMI - Fundo Monetário Internacional, a OMC Organização Mundial do Comércio, começaram a discutir os efeitos perversos e negativos da degradação social e ambiental. Estes organismos percebem um aumento substancial da convulsão social e destruição ambiental em larga escala, que passa a mostrar claramente, através de várias manifestações, a intolerância crescente frente a um quadro global cada vez mais desanimador.

Um estudo da consultoria KPMG em 2006, denominado Global Sustainability Services, pontuou que as emissões de gás carbônico (CO2) e outros gases poluentes está alterando rapidamente biomas e microclimas ao redor do planeta. O regime de monções no sudeste asiático, bem como as alterações nos regimes de cheia e baixa dos rios em algumas regiões afeta diretamente não só as populações, mas todo o sistema econômico de diversas nações.

No mesmo estudo, foi ilustrado um survey conduzido pelo IPCC - Intergovernmental Panel on Climate Change que apurou que a quantidade de $\mathrm{CO} 2$ na atmosfera aumentou $35 \%$ nos últimos 250 anos, excedendo em muito as variações naturais ocorridas nos 650.000 anos. Um relatório da UNICEF citado no estudo de Souza e Harb (2009) sobre água e saneamento ilustrou que mais de um milhão e meio de crianças menores de cinco anos morrem devido à escassez de água de qualidade e precárias condições de higiene.

Ainda segundo o estudo da KPMG (2006), os últimos verões no hemisfério norte, notadamente nos Estados Unidos e no Reino Unido, apresentaram temperaturas extremamente altas, com grandes danos à população e ao meio ambiente. Em uma série histórica de 1995 a 2006, os últimos 12 anos apresentaram abrupto crescimento nas temperaturas médias em todas as estações do ano.

Revista de Gestão Social e Ambiental - RGSA, São Paulo, v. 5, n. 3, p. 73-89, set./dez. 2011. 
Aligleri et al. (2009) pontuam que hoje a população mundial é de 6,7 bilhões de pessoas, sendo que, em 2050, a ONU estima mais 2,5 bilhões de pessoas, pessoas que utilizam os recursos naturais em larga escala para comer, morar, vestir, se aquecer e se deslocar. O uso exacerbado dos recursos não renováveis está comprometendo o equilíbrio do planeta e a vida humana. $O$ crescimento não significa mais, necessariamente, desenvolvimento. Segundo os autores, para que a sociedade progrida e se desenvolva, é preciso dar atenção a fatores que possam afetar a estabilidade futuramente.

Diante deste cenário, as discussões passaram a se centrar na questão do desenvolvimento sustentável, desenvolvimento este baseado no tripé econômico, social e ambiental, provendo melhores condições de vida para todos. Macedo e Cípola (2009) comentam que o reconhecimento dos problemas econômicos, sociais e ambientais não tratados foi o terreno fértil para a inserção da discussão sobre sustentabilidade.

Para Monzoni, Biderman e Brito (2006) o início do século XXI é marcado pelo início da discussão sobre a sustentabilidade. Diante das incertezas sobre o futuro e dado o crescente cenário de degradação ambiental, social e econômica, os debates começaram a se centrar em como prover ou mesmo enxergar soluções em um cenário tão caótico. No entendimento de Aligleri et al (2009), organizações não governamentais, governos, mídia e consumidores mais conscientes questionam o crescimento econômico a qualquer custo e pressionam a revisão deste cenário.

Aliglieri et al (2009) afirmam que a preocupação com posturas socialmente corretas, ambientalmente sustentáveis e economicamente viáveis está cada vez mais presente entre os temas de gestão. Neste sentido, a sustentabilidade pode ser percebida como um dos temas mais debatidos e propagados na gestão.

Ruthes e Nascimento (2006) ilustram que o desenvolvimento sustentável decorre da combinação equilibrada de desenvolvimento econômico com justiça social e domínio dos problemas ambientais. Para os autores, o desenvolvimento sustentável, então, se refere à sustentabilidade econômica, social e ambiental, sendo, portanto, uma integração destas perspectivas em uma única.

Para Vellani e Ribeiro (2006), estas dimensões, econômica, social e ambiental, se referem ao chamado Tripple Bottom Line (TBL) da sustentabilidade. Macedo (2009) afirma que o TBL reflete a atuação equlibrada no bottom line econômico, no bottom line social e no bottom line ambiental. Isto posto, se mantém: a sustentabilidade econômica, ao gerar riqueza; a sustentabilidade social ao estimular a educação, cultura, lazer e justiça social; e a sustentabilidade ambiental ao manter ecossistemas vivos, com diversidade e vida (Vellani \& Ribeiro, 2006).

Savitz (2007) argumenta que a sustentabilidade está se transformando rapidamente numa tendência dominante, pois as iniciativas sustentáveis evoluíram ao longo dos estágios de heresia, inviabilidade, idealismo e, finalmente, bom senso, em função do respeito ao equilíbrio entre as perspectivas ambiental, social e econômica. Macedo (2009) comenta que a amplitude e a importância do conceito de desenvolvimento sustentável fazem com que se torne necessário o desenvolvimento de sistemas de informação para apoiar o processo de gestão do mesmo, através da elaboração de metodologias para mensuração do nível de sustentabilidade.

Ainda segundo Macedo (2009), torna-se necessária a criação de um ferramental que auxilie no acompanhamento e na avaliação dos avanços e/ou retrocessos do desenvolvimento sustentável, por meio da utilização de um conjunto de indicadores relacionados à sustentabilidade, tornando-a mensurável e assim mais facilmente operacionalizada.

Aligleri et al (2009) ilustram que a questão do desenvolvimento sustentável ganha vulto na discussão entre os países membros das Nações Unidas. Este fato resulta em diretrizes que, de certa forma, orientam toda uma formulação conceitual. Estas diretrizes foram traduzidas em padrões, acordos e recomendações no âmbito das Nações Unidas e seus organismos, como a Organização Internacional do Trabalho (OIT) e o Programa das Nações Unidas para o Desenvolvimento 
(PNUD). Algumas iniciativas globais são: a Carta da Terra, o Protocolo de Kioto, o Pacto Global, as Metas do Milênio e os Princípios do Equador.

A Carta da Terra nasceu em 1997 como resposta às ameaças que se instalam sobre o planeta, pensando articuladamente os problemas ecológicos e sociais, pretendendo se equiparar à Declaração Internacional dos Direitos Humanos em termos de sustentabilidade, justiça econômica, ética e paz.

O Protocolo de Kioto é um tratado internacional firmado também em 1997 com compromissos sobre a redução global de gases que provocam o efeito estufa e o aquecimento global. Por este protocolo, se propõe um calendário no qual os países desenvolvidos têm a obrigação de reduzir a quantidade de gases poluentes, sendo que os planos de redução precisam ser colocados em prática até 2012.

O Pacto Global foi lançado em 1999, em parceria entre a ONU, o setor empresarial, a sociedade civil e organizações do setor público, fundamentando-se em dez princípios ligados a direitos humanos, relações de trabalho, meio ambiente e combate à corrupção, aliando negócios ao desenvolvimento sustentável.

O programa Metas do Milênio firmado em 2000 é um documento que consolida os chamados Objetivos de Desenvolvimento do Milênio (ODM) que devem ser alcançados até 2015 pelos países membros das Nações Unidas. Os oito objetivos são: erradicar a extrema pobreza e a fome; universalizar o ensino básico; promover a igualdade de gênero e autonomia das mulheres; reduzir a mortalidade infantil; melhorar a saúde materna; combater a AIDS, a malária e outras doenças; garantir a sustentabilidade ambiental; estabelecer uma cooperação universal para o desenvolvimento. A partir destes oito objetivos internacionais comuns, dezoito metas e quarenta e oito indicadores foram definidos para possibilitar uma avaliação uniforme nos níveis global, regional e nacional.

Finalmente os Princípios do Equador são estabelecidos pela International Finance Corporation (IFC), braço financeiro do Banco Mundial, para serem aplicados por instituições financeiras no financiamento de grandes projetos de investimentos, estabelecendo critérios para a concessão de crédito, assegurando que os projetos financiados sejam desenvolvidos de forma sustentável.

Lima et al. (2008), Barddal e Alberton (2008) e Macedo (2009) relatam a existência de vários modelos de indicadores de sustentabilidade, destacando três destes: Ecological Footprint Method, Barometer of Sustainability e Dashboard of Sustainability.

O Ecological Footprint Method permite estimar as exigências de consumo de recursos e a assimilação de resíduos de uma determinada população ou economia em termos de área correspondente de terra produtiva ou água, fundamentando-se no conceito de capacidade de carga. Assim sendo, revela a área de ecossistema necessária para assegurar a sobrevivência de uma determinada população. Para tanto, é preciso considerar as implicações (coeficientes técnicos) de cada tipo de consumo (alimentação, habitação, transporte, bens de consumo e serviço) em termos de demanda por recursos naturais.

Já o Barometer of Sustainability procura, através da consideração equilibrada das dimensões ambiental e social, efetuar a medição do progresso de uma localidade em direção à sustentabilidade, indicando, nesta configuração bidimensional, a situação da mesma, que varia desde circunstâncias ruins até boas em relação ao desenvolvimento sustentável.

Por fim, o Dashboard of Sustainability tem como objetivo medir a utilização de estoques e fluxos das dimensões do desenvolvimento sustentável (ambiental, social, econômica e institucional). Seus indicadores podem ser avaliados a partir de dois critérios: importância e desempenho. A visualização dos indicadores se dá graficamente, através de um painel de controle ilustrado a partes de uma escala de cores: vermelha para situação crítica, amarela para situação intermediária e verde para situação excelente. Este permite avaliar a performance de cada uma das dimensões individualmente ou de forma agregada. Estas características tornam esta ferramenta 
muito atrativa, principalmente em função da visão holística e da facilidade de interpretação dos resultados (comunicação visual).

Todos os elementos discutidos evidenciam a complexidade e a importância do conceito de desenvolvimento sustentável. Neste sentido, percebe-se a necessidade de construir mais ferramentas que mensurem o nível de sustentabilidade do processo de desenvolvimento de uma região (país, município, etc.).

É exatamente neste contexto que se propõe este trabalho, pois procura-se, através da aplicação da DEA, verificar o nível de sustentabilidade, de modo relativo e multicriterial, através do cálculo do Índice Consolidado de Desenvolvimento Sustentável (ICDS), que envolve as perspectivas ambiental, social e econômica, das unidades federativas do Brasil e de suas capitais.

\section{MÉTODO DE PESQUISA}

Esta pesquisa pode ser caracterizada, de acordo com Vergara (2009), como sendo descritiva e quantitativa, pois procura-se através da aplicação da Análise Envoltória de Dados (DEA) às informações das unidades federativas do Brasil e de suas capitais, expor características a respeito de seus níveis de sustentabilidade.

Foram obtidas informações sobre os Estados mais o Distrito Federal, num total de 27 UFs, e das capitais destes Estados, num total de 26 municípios.

Os dados utilizados, tanto para as UFs quanto para as capitais, são secundários e extraídos basicamente de três fontes:

- do Relatório sobre Produto Interno Bruto (PIB) dos Municípios Brasileiros publicado pelo Instituto Brasileiro de Geografia e Estatística (IBGE) foi obtida a proxy para a perspectiva econômica, que foi o PIBpercapita para o ano de 2006;

- do Atlas de Desenvolvimento Humano do Programa das Nações Unidas para o Desenvolvimento (PNUD) foi obtido o indicador da perspectiva social - o Índice de Desenvolvimento Humano Municipal (IDH-M) para o ano de $2000 \mathrm{e}$

- do Sistema de Coleta de Dados Contábeis de Estados e Municípios (SISTN) da Secretaria do Tesouro Nacional (STN) do Ministério da Fazenda foi obtido o indicador ambiental. Este representa, para o ano de 2008, o percentual das despesas com a função Gestão Ambiental (\%DGA) em relação às despesas totais.

Logo, a análise que será conduzida neste trabalho utiliza proxies das três principais dimensões da sustentabilidade, segundo o Triple Bottom Line (TBL), sendo o IDH-M para a perspectiva social, o percentual de gastos com Gestão Ambiental para a perspectiva ambiental e o PIB per capita para a perspectiva econômica.

Sendo assim, a lógica para a análise é que uma UF ou uma capital será melhor, em termos de nível de sustentabilidade, quão melhores forem estes três vetores do desenvolvimento sustentável. Cabe ressaltar que para viabilizar a comparabilidade das unidades sob análise são feitos estudos separados, sendo um para todas as 27 UFs e outro para todas as 26 capitais. Os quadros $01 \mathrm{e}$ 02 mostram um resumo destas informações para as UFs e para as capitais, respectivamente: 
Quadro 01: Resumo das Informações das UFs

\begin{tabular}{|l|r|r|r|}
\hline Informações & PIBpc 2006 (mil R\$) & \multicolumn{1}{|c|}{ IDHM - 2000 } & \% Gastos Amb (2008) \\
\hline Média & 10,678 & 0,738 & 0,473 \\
\hline DP & 6,658 & 0,057 & 0,295 \\
\hline Mediana & 8,543 & 0,735 & 0,448 \\
\hline Maior Valor & 37,599 & 0,844 & 1,216 \\
\hline Menor valor & 4,212 & 0,636 & 0,043 \\
\hline Quartil Superior & 12,747 & 0,777 & 0,625 \\
\hline Quartil Inferior & 6,640 & 0,699 & 0,226 \\
\hline
\end{tabular}

Em uma análise de desempenho, ressaltam Gomes, Gonzalez-Araya e Carignano (2004), pode-se utilizar uma análise monocritério ou uma multicritério. Na Análise Monocritério, que neste caso pode ser expressa por cada uma das perspectivas listadas, pode-se valorar cada ação potencial sobre um único eixo de significância, ou seja, uma unidade comum. Já na Análise Multicritério temse um novo paradigma que reconhece a heterogeneidade das dimensões de desempenho, através da utilização de uma família de múltiplos critérios, escolhidos segundo as exigências técnicas, inteligibilidade, aceitação como base do trabalho e facilidade de utilização como instrumento de análise.

Quadro 02: Resumo das Informações das Capitais

\begin{tabular}{|l|r|r|r|}
\hline Informações & PIBpc 2006 (mil R\$) & \multicolumn{1}{|c|}{ IDHM - 2000 } & \% Gastos Amb (2008) \\
\hline Média & 14,003 & 0,805 & 0,822 \\
\hline DP & 8,922 & 0,036 & 0,769 \\
\hline Mediana & 10,804 & 0,799 & 0,632 \\
\hline Maior Valor & 51,961 & 0,875 & 3,207 \\
\hline Menor valor & 7,468 & 0,739 & 0,002 \\
\hline Quartil Superior & 15,654 & 0,837 & 1,236 \\
\hline Quartil Inferior & 8,954 & 0,778 & 0,190 \\
\hline
\end{tabular}

Neste artigo, para consolidar o nível de sustentabilidade, num índice denominado Índice Consolidado de Desenvolvimento Sustentável (ICDS), utiliza-se uma ferramenta de Análise Multicritério: a Análise Envoltória de Dados (DEA). Com esta é possível avaliar o nível de sustentabilidade de cada UF e de cada Capital de modo multicriterial, ou seja, considerando de maneira integrada todos os vetores do desenvolvimento sustentável apresentados (ambiental, social e econômico).

De acordo com Charnes, Cooper, Lewin e Seiford (1994) e Coelli, Rao e Baltese (1998), a Análise Envoltória de Dados (DEA), mostra o quão uma unidade é eficiente, no tratamento de seus inputs e outputs, em relação às outras, numa análise que fornece um indicador que varia de 0 a 1 ou de $0 \%$ a $100 \%$. Somente as unidades que obtêm índice de eficiência igual a um é que fazem parte da fronteira eficiente.

Segundo Cooper, Seiford e Zhu (2004) a metodologia DEA tem sua origem com o trabalho de Farrell (1957), que propôs uma abordagem de análise que pudesse ser mais adequada à gestão de qualquer organização produtiva. Já os modelos DEA, propriamente ditos, tiveram seu início em 1978 com a tese de Ph.D de Edward Rhodes sob a orientação de W.W. Cooper. Em seu trabalho, Rhodes ampliou o trabalho de Farrell analisando unidades que possuíam mais de um input e/ou output.

A metodologia DEA foi inicialmente desenvolvida no modelo de Retornos Constantes de Escala (CRS - Constant Returns to Scale), também conhecido por CCR (Charnes, Cooper \& Rhodes, 1978). Este modelo determina uma fronteira CRS que indica que crescimento proporcional dos inputs produzirá crescimento proporcional dos outputs. Este modelo tem como propriedades a 
convexidade, cálculo da ineficiência e o raio ilimitado (que presume a proporcionalidade entre inputs e outputs).

Depois, em 1984, foi desenvolvido o modelo BCC (Banker, Charnes \& Cooper, 1984) ou VRS (Variable Returns to Scale), que assume rendimentos crescentes e decrescentes de escala na fronteira de eficiência. Este modelo surgiu como resultante da partição da eficiência do modelo CCR em duas componentes: a eficiência técnica (VRS) e a eficiência de escala (CRS/VRS).

No caso das formulações da DEA, além das da escolha entre CRS e VRS existe a necessidade de fixação da ótica de análise (orientação input ou orientação output). Macedo, Cípola e Ferreira (2008) dizem que a abordagem DEA baseada nas entradas (inputs) busca maximizar as quantidades de produtos, isto é, maximizar uma combinação linear das quantidades dos vários produtos da unidade sob análise. Já para uma abordagem baseada nas saídas (outputs), busca-se minimizar as quantidades de insumos, isto é, minimizar uma combinação linear das quantidades dos vários insumos.

A Análise Envoltória de Dados (DEA), segundo Lins e Meza (2000) e Soares de Mello, Ângulo-Meza, Gomes e Biondi (2005), apresenta-se como uma metodologia matemática nãoparamétrica, baseada em programação linear, que fornece uma medida de desempenho capaz de comparar a eficiência de várias unidades similares e homogêneas, as DMU's (Decision Making Units), mediante a consideração explícita do uso de suas múltiplas entradas para a produção de múltiplas saídas. Desta forma, esta metodologia faz com que a decisão fique orientada por um único indicador construído a partir de várias abordagens de desempenho diferentes, através da relação ponderada entre inputs e outputs.

Apesar de originalmente ser uma metodologia proposta em um ambiente de produção (transformação de insumos em produtos), salientam Macedo e Cípola (2009), a DEA pode ser utilizada como um método multicritério, quando se utiliza indicadores do tipo quanto menor melhor no lugar dos inputs (ex.: risco, custo, endividamento etc.) e do tipo quanto maior melhor no lugar dos outputs (ex.: lucratividade, retorno, liquidez etc.). Isso transforma a DEA em um método de Apoio Multicritério à Decisão (AMD), aplicado com o intuito de consolidar várias perspectivas (critérios) de desempenho diferentes.

Lins e Meza (2000) ressaltam que um caminho intuitivo para introduzir a DEA é por meio de forma de razão. Para cada DMU, procura-se obter uma medida de razão de todos os outputs sobre todos os inputs. Ou seja, a modelagem procura encontrar os pesos ótimos $\mathrm{u}_{\mathrm{j}}$ e $\mathrm{v}_{\mathrm{i}}$ para a resolução do seguinte problema de programação matemática.

$$
\begin{aligned}
\text { Max } E_{c}= & \frac{\sum_{j=1}^{s} u_{j} y_{j c}}{\sum_{i=1}^{m} v_{i} x_{i c}} \\
\text { S.a.: } \quad & \frac{\sum_{j=1}^{s} u_{j} y_{j k}}{\sum_{i=1}^{m} v_{i} x_{i k}} \leq 1, k=1,2, \ldots, c, \ldots, n \\
& u_{j} \geq 0, \forall j, \\
& v_{i} \geq 0, \forall i
\end{aligned}
$$

Neste estudo foi utilizado o modelo DEA-VRS-I. A escolha do modelo VRS se deu pelo fato de ter-se unidades de tamanhos muito diferentes, o que recomenda uma modelagem com retornos variáveis de escala. Já a orientação input se deu pelo objetivo de aumentar (maximizar) os indicadores de sustentabilidade. Em termos práticos, de acordo com Santos e Casa Nova (2005), o modelo procura identificar a eficiência de uma unidade comparando-a com os melhores 
desempenhos observados, através da resolução do seguinte problema de programação linear (PPL), que já considera a proposta de Charnes e Cooper (1962), para linearizar a relação apresentada anteriormente, que era um problema de programação fracionária.

De acordo com Macedo e Cípola (2009), neste modelo, c é a unidade (DMU - Decision Making Units) que está sendo avaliada e u' é uma variável irrestrita, que responde pela questão dos retornos variáveis de escala. $\mathrm{O}$ problema acima envolve a procura de valores para $\mathrm{u}_{\mathrm{j}} \mathrm{e} \mathrm{v}_{\mathrm{i}}$, que são os pesos, de modo que maximize a soma ponderada dos outputs $\left(\mathrm{y}_{\mathrm{j}}\right)$ da DMU em estudo, sujeita as restrições de que a soma ponderada dos inputs $\left(\mathrm{x}_{\mathrm{i}}\right)$, desta mesma DMU, seja igual a um e a diferença entre a soma ponderada dos outputs $\left(\mathrm{y}_{\mathrm{j}}\right)$ e a soma ponderada dos inputs $\left(\mathrm{x}_{\mathrm{i}}\right)$ seja menor ou igual a zero, para todas as DMU's. Esta última restrição faz com que quando o mesmo conjunto de coeficientes de entrada e saída (os vários $\mathrm{u}_{\mathrm{j}} \mathrm{e} \mathrm{v}_{\mathrm{i}}$ ) for aplicado a todas as outras unidades que estão sendo comparadas, nenhuma unidade excederá $100 \%$ de eficiência.

$$
\begin{aligned}
\operatorname{Max} E_{c}= & \sum_{j=1}^{s} u_{j} y_{j c}+u^{\prime} \\
\text { S.a.: } \quad & \sum_{i=1}^{m} v_{i} x_{i c}=1 \\
& \sum_{j=1}^{s} u_{j} y_{j k}-\sum_{i=1}^{m} v_{i} x_{i k}+u^{\prime} \leq 0, k=1,2, \ldots, c, \ldots, n \\
& u_{j,} v_{i} \geq 0, \forall i, j .
\end{aligned}
$$

A modelagem DEA foi utilizada com um artifício matemático que busca melhorar o poder discriminante do modelo. Este artifício insere na análise uma fronteira invertida, que calcula a eficiência colocando os outputs no lugar dos inputs e os inputs, no lugar dos outputs. Ou seja, procura-se maximizar inputs/outputs. Tem-se, então, duas fronteiras de eficiência: a fronteira padrão DEA-VRS e uma fronteira invertida.

A fronteira invertida representa, segundo Macedo et al. (2008), uma visão pessimista de cada DMU, ou seja, torna nítido o que cada uma tem de pior, diferentemente da fronteira padrão ou otimista, que ao maximizar a eficiência acaba por ressaltar o que de melhor cada DMU possui, atribuindo, para tanto, peso zero às variáveis com comportamento ruim, podendo causar, assim, um grande número de unidades eficientes.

Logo, o desempenho calculado é função da proximidade de cada DMU em relação à fronteira otimista (padrão) e do distanciamento desta em relação à fronteira pessimista (invertida). O índice composto, então, é calculado pela média entre a eficiência padrão e o complemento da eficiência invertida (1 - Eficiência Invertida), alcançando valores que vão de zero a um (Macedo $e t$ al., 2008).

Cabe ressaltar que nesta análise, diferentemente do que é necessário, tem-se três indicadores de output (índices do tipo quanto maior, melhor). Ou seja, para aplicar a DEA faltaria a composição de pelos menos um indicador de input (índice do tipo quanto menor, melhor). Para solucionar este problema utilizou-se de três modelos DEA:

- Modelo 01: Tem como foco a perspectiva ambiental, pois a mesma terá seu valor invertido (1/\%DGA) e será considerado nesta análise como um input, permanecendo do mesmo jeito as outras duas variáveis (PIBpercapita e IDH-M). Este modelo integra os vetores de desenvolvimento sustentável, porém com foco na perspectiva ambiental, já que o modelo tem orientação input e foi esta a variável transformada em input.

- Modelo 02: Tem foco na perspectiva social, pois desta vez o input escolhido foi a inversão dos valores do IDH-M (1 - IDH-M). No restante possui as mesmas características do modelo 01. 
- Modelo 03: Seu foco é na perspectiva econômica, já que o input utilizado foi 1/PIBpercapita. As variáveis ambiental e social não sofreram alterações.

Assim sendo, tem-se três resultados para o ICDS: um maximizando as perspectivas social e econômica, dado a perspectiva ambiental (modelo 01); outro maximizando as perspectivas ambiental e econômica, dado a perspectiva social (modelo 02); e um último maximizando as perspectivas ambiental e social, dado a perspectiva econômica (modelo 03). Uma análise conjunta dos ICDS das UFs e das Capitais nestas três perspectivas (ambiental, social e econômica) mostra o nível de desenvolvimento sustentável de cada uma destas. No final obteve-se um ICDS final pela média dos ICDS de cada modelo.

Em cada um destes modelos, utilizou-se uma restrição aos pesos dos outputs, para se obter importâncias balanceadas/equilibradas $\left(\mathrm{u}_{\mathrm{j}}=50 \%\right)$. Ou seja, utilizou-se o artifício matemático de limitar ou restringir a atribuição de pesos pelo modelo, de modo que cada perspectiva ficasse no final com o mesmo peso/participação no Índice Consolidado de Desempenho Sustentável (ICDS).

Cabe ressaltar, que cada um destes modelos representa uma análise do nível de sustentabilidade multicriterial, consolidando os índices representativos das dimensões ambiental, social e econômica. Porém, cada um deles foi aplicado com um foco de consolidação diferente. Ou seja, cada um representa uma análise multicriterial do desenvolvimento sustentável (considerando todos os vetores da sustentabilidade), porém com foco principal em um dos elementos.

Para ter índice máximo (ICDS $=100 \%$ ), em cada modelo, a UF ou a Capital terá que possuir uma relação entre sustentabilidade ambiental, social e econômica ótima. Não adianta ter destaque em apenas um dos aspectos, isso no máximo vai dar destaque para o país em um dos modelos. A idéia é ter uma conjugação equilibrada, que mostre desenvolvimento econômico, com igualdade social e equilíbrio com o meio-ambiente.

Uma das características da DEA, segundo Macedo e Cípola (2009), é ser uma medida relativa de desempenho, ou seja, os scores verificados em determinada função somente valem para a amostra em estudo. Caso haja alteração na amostra com inclusão ou exclusão de novas DMU's ou alteração nas variáveis, torna-se necessário calcular novos scores de eficiência. Em síntese, os resultados de eficiência de determinada amostra não são comparáveis com os resultados de amostras diferentes e nem da população. É importante ressaltar, de acordo com Macedo (2009), que esta é uma característica não-paramétrica da metodologia DEA, em que os parâmetros de desempenhos são reais e estabelecidos dentro da amostra, levando em conta as variáveis sob análise.

Por fim, os modelos de análise, um para cada perspectiva do desenvolvimento sustentável, foram executados em um software específico de DEA, apresentado por Meza, Biondi, Soares de Mello, Gomes e Coelho (2003), denominado SIAD (Sistema Integrado de Apoio à Decisão).

\section{APRESENTAÇÃO E ANÁLISE DOS RESULTADOS}

De posse das informações de cada uma das UFs e das Capitais sob análise, no que diz respeito às variáveis de sustentabilidade ambiental, social e econômica, procedeu-se a aplicação da DEA, modelando-se os dados nestas três perspectivas (modelos 01, 02 e 03). Os quadros 03 e 04 mostram os resultados do Índice Consolidado de Desenvolvimento Sustentável (ICDS) para cada UF e para cada Capital, em cada perspectiva, respectivamente. Um ponto que deve ser destacado na análise que gerou estes quadros é o fato de nenhuma das unidades sob análise atingir a eficiência máxima de $100 \%$. Isso acontece por decisão dos pesquisadores, que utilizam na análise uma metodologia com base em DEA que resulta num indicador, entre 0 e 1 , mas que dificilmente chega ao máximo de $100 \%$. O valor máximo para o indicador de eficiência não é atingido por conta da pertinência que cada DMU tem em relação às duas fronteiras. Logo, se existe alguma pertinência em relação a fronteira invertida isso fará com que o indicador final seja necessariamente diferente de $100 \%$. 
Numa análise dos resultados do quadro 03, que mostra o ICDS em suas três perspectivas (modelos) para as UFs, pode-se perceber que as UFs com destaque positivo em relação à perspectiva ambiental são DF, MG e RN. Observa-se que todas estas UFs possuem indicador nesta perspectiva superior a $90 \%$.

Já em relação ao enfoque social tem-se como destaque positivo apenas o DF, que mesmo sendo o de maior desempenho tem indicador de apenas 78,57 \%. Por fim, na perspectiva econômica observa-se que o DF e SP são os destaques positivos com indicadores superiores a $80 \%$, sendo que o DF atinge mais de 90 \% de eficiência econômica.

Quadro 03: Resultado do ICDS para as UFs nas três perspectivas

\begin{tabular}{|c|c|c|c|c|c|c|c|}
\hline UF & Ambiental & Social & Econômica & UF & Ambiental & Social & Econômica \\
\hline AC - ACRE & $77,70 \%$ & $34,12 \%$ & $41,04 \%$ & PB - PARAÍBA & $77,91 \%$ & $26,44 \%$ & $29,10 \%$ \\
\hline AL-ALAGOAS & $22,65 \%$ & $22,86 \%$ & $10,53 \%$ & PE - PERNAMBUCO & $38,00 \%$ & $33,84 \%$ & $20,64 \%$ \\
\hline AM - AMAZONAS & $78,83 \%$ & $37,75 \%$ & $56,72 \%$ & PI - PIAUI & $14,73 \%$ & $22,67 \%$ & $5,60 \%$ \\
\hline AP - AMAPÁ & $38,02 \%$ & $40,00 \%$ & $24,95 \%$ & PR - PARANÁ & $70,56 \%$ & $57,36 \%$ & $51,48 \%$ \\
\hline BA - BAHIA & $62,06 \%$ & $32,14 \%$ & $28,76 \%$ & RJ - RIO DE JANEIRO & $81,09 \%$ & $63,90 \%$ & $67,48 \%$ \\
\hline CE - CEARÁ & $63,42 \%$ & $34,79 \%$ & $20,40 \%$ & RN - RIO GRANDE DO NORTE & $96,50 \%$ & $35,92 \%$ & $68,81 \%$ \\
\hline DF - DISTRITO FEDERAL & $95,38 \%$ & $78,57 \%$ & $94,40 \%$ & RO - RONDÔNIA & $71,50 \%$ & $43,03 \%$ & $38,68 \%$ \\
\hline ES - ESPÍRITO SANTO & $57,93 \%$ & $50,91 \%$ & $55,45 \%$ & RR - RORAIMA & $26,13 \%$ & $31,67 \%$ & $19,46 \%$ \\
\hline GO - GOIÁS & $2,14 \%$ & $34,82 \%$ & $13,24 \%$ & RS - RIO GRANDE DO SUL & $53,23 \%$ & $65,16 \%$ & $51,74 \%$ \\
\hline MA - MARANHÃO & $9,98 \%$ & $21,43 \%$ & $6,15 \%$ & SC - SANTA CATARINA & $37,04 \%$ & $60,88 \%$ & $47,71 \%$ \\
\hline MG-MINAS GERAIS & $91,16 \%$ & $53,18 \%$ & $79,42 \%$ & SE - SERGIPE & $89,49 \%$ & $30,85 \%$ & $60,71 \%$ \\
\hline MS - MATO GROSSO DO SUL & $51,51 \%$ & $53,34 \%$ & $40,92 \%$ & SP - SÃO PAULO & $87,91 \%$ & $68,61 \%$ & $83,55 \%$ \\
\hline MT - MATO GROSSO & $86,33 \%$ & $53,18 \%$ & $72,40 \%$ & TO - TOCANTINS & $56,33 \%$ & $37,06 \%$ & $30,37 \%$ \\
\hline PA - PARÁ & $53,07 \%$ & $40,11 \%$ & $24,55 \%$ & & & & \\
\hline
\end{tabular}

Como destaque negativo, em relação à perspectiva ambiental, tem-se GO e MA, ambos com indicadores inferiores a $10 \%$. Já na perspectiva social percebe-se que o MA apresenta o menor desempenho, mesmo sendo este um pouco superior a $20 \%$. Na perspectiva econômica percebe-se que MA e PI são os destaques negativos, ambos com menos e $10 \%$ de eficiência econômica.

Percebe-se, então, que o DF possui destaque positivo nas três perspectivas, enquanto que MA é tido como destaque negativo nestes três enfoques da sustentabilidade. Isso mostra uma posição de superioridade para o DF, que coloca esta UF num patamar de destaque em termos de desenvolvimento sustentável. Mas por outro lado mostra uma situação preocupante para o MA, pois existe uma situação problemática de sustentabilidade em todas as perspectivas.

Já a análise dos resultados para as 26 capitais (quadro 04) mostra que tem-se como destaque positivo em termos de vertente ambiental Vitória no ES, que possui indicador próximo de $100 \%$. Para a perspectiva social tem-se como destaques positivos os municípios de Vitória-ES, Porto Alegre-RS e Florianópolis-SC, mesmo que todos com indicadores apenas um pouco superiores a 70 $\%$. Por fim, no enfoque econômico tem-se destaque positivo apenas para Vitória-ES com indicador acima de $90 \%$. 
Quadro 04: Resultado do ICDS para as Capitais nas três perspectivas

\begin{tabular}{|c|c|c|c|c|c|c|c|}
\hline UF - Capital & Ambiental & Social & Econômica & UF - Capital & Ambiental & Social & Econômica \\
\hline AC - RIO BRANCO & $75,41 \%$ & $28,28 \%$ & $11,81 \%$ & PB - JOÃO PESSOA & $54,17 \%$ & $37,23 \%$ & $16,50 \%$ \\
\hline AL- MACEIÓ & $0,84 \%$ & $23,95 \%$ & $7,58 \%$ & PE - RECIFE & $58,20 \%$ & $41,90 \%$ & $30,75 \%$ \\
\hline AM - MANAUS & $62,04 \%$ & $34,76 \%$ & $48,30 \%$ & PI - TERESINA & $2,46 \%$ & $26,71 \%$ & $7,19 \%$ \\
\hline AP - MACAPÁ & $69,35 \%$ & $33,73 \%$ & $17,91 \%$ & PR - CURITIBA & $65,40 \%$ & $66,67 \%$ & $46,53 \%$ \\
\hline BA - SALVADOR & $46,12 \%$ & $42,91 \%$ & $11,26 \%$ & RJ - RIO DE JANEIRO & $69,49 \%$ & $60,59 \%$ & $52,18 \%$ \\
\hline CE - FORTALEZA & $85,67 \%$ & $38,25 \%$ & $18,48 \%$ & RN - NATAL & $53,66 \%$ & $38,87 \%$ & $19,15 \%$ \\
\hline ES - VITÓRIA & $99,98 \%$ & $72,41 \%$ & $92,81 \%$ & RO - PORTO VELHO & $55,38 \%$ & $30,97 \%$ & $21,70 \%$ \\
\hline GO - GOIANIA & $70,79 \%$ & $55,50 \%$ & $33,87 \%$ & RR - BOA VISTA & $51,91 \%$ & $35,94 \%$ & $24,37 \%$ \\
\hline MA - SÃO LUIS & $50,85 \%$ & $35,62 \%$ & $26,78 \%$ & RS - PORTO ALEGRE & $68,55 \%$ & $71,88 \%$ & $52,26 \%$ \\
\hline MG - BELO HORIZONTE & $78,12 \%$ & $58,75 \%$ & $35,42 \%$ & SC - FLORIANÓPOLIS & $57,36 \%$ & $76,05 \%$ & $42,92 \%$ \\
\hline MS - CAMPO GRANDE & $56,65 \%$ & $47,97 \%$ & $23,28 \%$ & SE - ARACAJU & $0,02 \%$ & $30,34 \%$ & $10,73 \%$ \\
\hline MT - CUIABÁ & $48,69 \%$ & $48,52 \%$ & $30,32 \%$ & SP - SÃO PAULO & $62,34 \%$ & $59,80 \%$ & $60,17 \%$ \\
\hline PA - BELÉM & $66,85 \%$ & $45,05 \%$ & $15,83 \%$ & TO - PALMAS & $61,07 \%$ & $42,94 \%$ & $15,76 \%$ \\
\hline
\end{tabular}

Em termos de destaque negativo percebe-se que na perspectiva ambiental os municípios de Maceió-AL e Aracajú-SE tem indicadores inferiores a $1 \%$. Já no que diz respeito ao enfoque social observa-se que Maceió-AL novamente aparece como o município de pior desempenho, mesmo que um pouco superior a $20 \%$. Por último, a análise da perspectiva econômica mostra que Maceió-AL e Teresina-PI são as capitais de pior desempenho, com índices abaixo de $10 \%$.

Com este quadro de desempenho pode-se dizer que Vitória-ES aparece como grande destaque positivo, enquanto Maceió-AL se mostra com problemas em todas as perspectivas. Isso mostra uma condição especial de sustentabilidade para Vitória-ES e uma situação preocupante em todas as perspectivas para Maceió-AL.

Feita esta análise por perspectiva pode-se partir para a integração dos indicadores encontrados para cada uma destas. Isso foi feito pela média entre os indicadores para cada UF e para cada Capital. Os quadros 05 e 06 mostram os resultados, já em ordem decrescente do ICDS, para as UFs e para as Capitais, respectivamente.

Quadro 05: Resultado do ICDS Final para as UFs

\begin{tabular}{|l|c|l|c|}
\hline \multicolumn{1}{|c|}{ UF } & ICDS & \multicolumn{1}{c|}{ UF } & ICDS \\
\hline DF - DISTRITO FEDERAL & $89,45 \%$ & SC - SANTA CATARINA & $48,54 \%$ \\
\hline SP - SÃO PAULO & $80,02 \%$ & PB - PARAÍBA & $44,48 \%$ \\
\hline MG - MINAS GERAIS & $74,59 \%$ & TO - TOCANTINS & $41,25 \%$ \\
\hline RJ - RIO DE JANEIRO & $70,82 \%$ & BA - BAHIA & $40,99 \%$ \\
\hline MT - MATO GROSSO & $70,64 \%$ & CE - CEARÁ & $39,54 \%$ \\
\hline RN - RIO GRANDE DO NORTE & $67,08 \%$ & PA - PARÁ & $39,24 \%$ \\
\hline SE - SERGIPE & $60,35 \%$ & AP - AMAPÁ & $34,33 \%$ \\
\hline PR - PARANÁ & $59,80 \%$ & PE - PERNAMBUCO & $30,83 \%$ \\
\hline AM - AMAZONAS & $57,77 \%$ & RR - RORAIMA & $25,75 \%$ \\
\hline RS - RIO GRANDE DO SUL & $56,71 \%$ & AL - ALAGOAS & $18,68 \%$ \\
\hline ES - ESPÍRITO SANTO & $54,77 \%$ & GO - GOIÁS & $16,73 \%$ \\
\hline RO - RONDÔNIA & $51,07 \%$ & PI - PIAUI & $14,34 \%$ \\
\hline AC - ACRE & $50,96 \%$ & MA - MARANHÃO & $12,52 \%$ \\
\hline MS - MATO GROSSO DO SUL & $48,59 \%$ & & \\
\hline
\end{tabular}

Como já era esperado o quadro 05 mostra o DF como a UF de melhor desempenho. Esta é seguida por SP, MG, RJ e MT, todas com ICDS superiores a $70 \%$. O quadro também mostra o desempenho problemático do MA, que tem o pior ICDS. Este é acompanhado por outras UFs que possuem também desempenho inferior a $20 \%$, tais como PI, GO e AL. 
Isso quer dizer que o DF é a UF, considerando as variáveis do modelo, de melhor nível de desenvolvimento sustentável, em todas as perspectivas combinadas: ambiental (modelo 01), social (modelo 02) e econômica (modelo 03). Esta é a UF que melhor conjuga os indicadores considerados, minimizando inputs e maximizando outputs, em todos os modelos. Assim sendo, pode-se dizer que o DF possui um equilíbrio, em termos ambiental, social e econômico, que colocao numa posição de destaque em termos de sustentabilidade.

Já o bom resultado das outras UFs se deve ao fato de terem tido desempenho bom em praticamente todas as perspectivas, mesmo não aparecendo como destaque positivo na análise anterior a conjugação dos indicadores levou estas UFs a uma posição final de destaque.

Já o MA apresenta os piores resultados em todas as perspectivas, culminando numa consolidação ruim dos indicadores de sustentabilidade. Para as outras UFs com as piores colocações no ranking percebe-se que as mesmas possuem desempenhos ruins em todas as perspectivas, sendo inclusive apontadas como destaques negativos na análise anterior.

Já a análise do quadro 06 mostra esta mesma situação de consolidação das perspectivas de sustentabilidade, porém desta vez para as capitais. Mas os resultados também estão dentro do que se esperava dado as análises conduzidas para cada perspectiva.

Quadro 06: Resultado do ICDS Final para as Capitais

\begin{tabular}{|l|c|l|c|}
\hline \multicolumn{1}{|c|}{ UF - Capital } & ICDS & \multicolumn{1}{c|}{ UF - Capital } & ICDS \\
\hline ES - VITÓRIA & $88,40 \%$ & MT - CUIABÁ & $42,51 \%$ \\
\hline RS - PORTO ALEGRE & $64,23 \%$ & AP - MACAPÁ & $40,33 \%$ \\
\hline SP - SÃO PAULO & $60,77 \%$ & TO - PALMAS & $39,92 \%$ \\
\hline RJ - RIO DE JANEIRO & $60,75 \%$ & AC - RIO BRANCO & $38,50 \%$ \\
\hline PR - CURITIBA & $59,53 \%$ & MA - SÃO LUIS & $37,75 \%$ \\
\hline SC - FLORIANÓPOLIS & $58,78 \%$ & RR - BOA VISTA & $37,41 \%$ \\
\hline MG - BELO HORIZONTE & $57,43 \%$ & RN - NATAL & $37,23 \%$ \\
\hline GO - GOIANIA & $53,39 \%$ & RO - PORTO VELHO & $36,02 \%$ \\
\hline AM - MANAUS & $48,37 \%$ & PB - JOÃO PESSOA & $35,97 \%$ \\
\hline CE - FORTALEZA & $47,47 \%$ & BA - SALVADOR & $33,43 \%$ \\
\hline PE - RECIFE & $43,62 \%$ & SE - ARACAJU & $13,70 \%$ \\
\hline MS - CAMPO GRANDE & $42,63 \%$ & PI - TERESINA & $12,12 \%$ \\
\hline PA - BELÉM & $42,58 \%$ & AL - MACEIÓ & $10,79 \%$ \\
\hline
\end{tabular}

A cidade de Vitória-ES aparece como o grande destaque positivo e até com certa folga em relação às outras capitais, já que possui ICDS de quase $90 \%$, enquanto que a segunda colocada que é Porto Alegre-RS possui um índice apenas pouco superior a $60 \%$. Este era um resultado esperado dado o grande destaque positivo que Vitória-ES teve na análise de cada perspectiva separadamente.

Do outro lado do ranking tem-se Maceió-AL, Teresina-PI e Aracajú-SE como as capitais de pior desempenho consolidado. Isso quer dizer que estas capitais não têm uma situação que aponte para uma situação de desenvolvimento econômico com crescimento humano e equilíbrio ecológico. Esta situação também já era esperada, pois estes são os municípios apontados como destaques negativos na análise das perspectivas feita anteriormente.

Por fim, um ponto que chama atenção na análise dos dados é a observação da dispersão das unidades analisadas em cada perspectiva. As vertentes ambiental e econômica possuem uma dispersão muito maior do que o enfoque social. Isso pode ser visto pela análise os índices nos quadros 03 e 04 ou até mesmo na análise das variáveis nos quadros 1 e 2 . Isso quer dizer que as UFs e as Capitais possuem uma diferenciação muito maior no que diz respeito a atuação ambiental e o desenvolvimento econômico, do que nas condições para o crescimento humano. 


\section{CONSIDERAÇÕES FINAIS}

Esta pesquisa teve como objetivo analisar o nível de sustentabilidade de cada UF e de cada Capital no contexto brasileiro. A análise, para alcançar este objetivo, se deu pela aplicação da Análise Envoltória de Dados (DEA), como uma ferramenta de apoio multicritério, que consolidou as diversas perspectivas do desenvolvimento sustentável (ambiental, social e econômica) em um único indicador. Cabe ressaltar, que isso facilita o processo de análise/avaliação, pois, ao invés de considerar vários índices para concluir a respeito da sustentabilidade, utiliza-se apenas da medida agregada de eficiência da DEA.

Os resultados desse estudo como um todo, propõem uma nova percepção sobre o nível de sustentabilidade de localidades, que não se encontra disponível através de nenhuma das outras técnicas de análise do desenvolvimento sustentável. Isto porque apresenta um único indicador, que conjuga todas as perspectivas relevantes, de modo multicriterial e relativo. Ou seja, a partir de informações que não estariam disponíveis pelas técnicas listadas anteriormente, os resultados da Análise Envoltória de Dados (DEA) podem proporcionar melhores condições de análise/avaliação da sustentabilidade.

Cabe ressaltar, a importância de ferramentas como esta num contexto de preocupação mundial com o desenvolvimento sustentável, em que os governantes e a sociedade como um todo são cobrados, permanentemente, a terem atitudes positivas em relação ao desenvolvimento econômico concomitante com justiça social e respeito ao meio-ambiente.

De maneira geral, os resultados mostram que o DF e Vitória-ES são os que alcançaram os melhores níveis de sustentabilidade em todos os modelos (perspectivas), sendo considerados os de melhor nível de desenvolvimento sustentável, em todas as perspectivas conjugadas: ambiental (modelo 01), social (modelo 02) e econômica (modelo 03). Do outro lado do ranking de sustentabilidade aparecem MA e Maceió-AL, como as localidades problemáticas sob praticamente todas as perspectivas no que diz respeito ao desenvolvimento sustentável.

Uma questão relevante, que surge da comparação destes rankings é a posição dos estados de Alagoas e do Piauí, que possuem enquanto UFs desempenhos ruins e que têm suas capitais também com baixo nível de sustentabilidade. Esta é realmente uma situação preocupante e que merece uma intervenção urgente, com o desenvolvimento de políticas públicas que envolve as instâncias municipal, estadual e federal.

Por fim, cabe ressaltar que este estudo é apenas uma tentativa de aplicar a DEA para análise de sustentabilidade de regiões (países, municípios, etc.). Este deve ter continuidade procurando melhorar a qualidade informacional dos índices, utilizando dados menos agregados, como, por exemplo, a utilização dos índices que compõem o IDH ao invés dele já consolidado. Isso pode trazer luz para outras questões que se perdem com a agregação antecipada dos indicadores à aplicação da DEA. Num estudo com variáveis menos agregadas, pode-se pensar em aplicar outras técnicas para auxiliar na análise do nível de sustentabilidade, tais como as de Análise Multivariada (análise de conglomerados, análise discriminante, regressão múltipla, dentre outras) e os testes de hipóteses (para testar hipóteses de diferença no nível de sustentabilidade de certos grupos de localidades).

\section{REFERÊNCIAS}

Aligleri, L., Aligleri, L. A., \& Krugliankas, I. (2009). Gestão Socioambiental: responsabilidade e sustentabilidade do negócio. São Paulo: Atlas.

Banker, R. D., Charnes, A., \& Cooper, W. W. (1984). Some Models for Estimating Technical and Scale Inefficiencies in Data Envelopment Analysis. Management Science, 30(9), 1078-1092. 
Barddal, R., \& Alberton, A. (2008). Uma Análise Comparativa de Métodos de Mensuração da Sustentabilidade: aplicabilidade no setor turístico. In: SIMPÓSIO DE ADMINISTRAÇÃO DA PRODUÇÃO, LOGÍSTICA E OPERAÇÕES INTERNACIONAIS, 11, 2008. Anais do XI SIMPOI. São Paulo: FGV/EAESP. CD-ROOM.

Charnes, A., \& Cooper, W. W. (1962). Programming with Linear Fractional Functionals. Naval Research Logistic Quarterly, 9, 181-186.

Charnes, A., Cooper, W. W., Lewin, A. Y., \& Seiford, L. M. (1994). Data Envelopment Analysis. 2. ed. Boston: KAP.

Charnes, A., Cooper, W. W., \& Rhodes, E. (1978). Measuring the Efficiency of Decision Making Units. European Journal of Operational Research, 2(6), 429-444.

Coelli, T., Rao, D. S. P., Baltese, G. E. (1998). An Introduction to Efficiency and Productivity Analysis. Boston: KAP.

Cooper, W. W., Seiford, L. M., \& Zhu, J. (2004). Handbook on Data Envelopment Analysis. Boston: KAP.

Costanza, R. (1991). Ecological Economics: the science and management of sustainability. New York: Columbia Press.

Farrell, M. J. (1957). The Measurement of Productive Efficiency. Journal of the Royal Statistical Society. 120(A-3), 253-290.

Gomes, L. F. A. M., Gonzalez-Araya, M. C., \& Carignano, C. (2004). Tomada de decisões em cenários complexos. Rio de Janeiro: Pioneira Thompson Learning.

Grayson, D., \& Hodges, A. (2002). Compromisso social e gestão empresarial. São Paulo: Publifolha.

Jacobi, P. (1994). O Complexo Desafio da Sustentabilidade: desenvolvimento e meio ambiente. São Paulo: Nobel.

KPMG. (2006). Reporting the Business Impliacations of Climate Change in Sustainability Reports. A survey conducted by the Global Reporting Iniative and KPMG's Global Sustainability Services. Netherlands.

Lima, M. T. A., Gómez, C. R. P., \& Castillo, L. A. G. (2008). Cenários de Sustentabilidade: uma ferramenta para inserção do desenvolvimento sustentável na estratégia empresarial. In: SIMPÓSIO DE ADMINISTRAÇÃO DA PRODUÇÃO, LOGÍSTICA E OPERAÇÕES INTERNACIONAIS, 11, 2008. Anais do XI SIMPOI. São Paulo: FGV/EAESP. CD-ROOM.

Lima, P. R. S. (2005). Responsabilidade social: a experiência do Selo Empresa Cidadã na cidade de São Paulo. São Paulo: Educ-Fapesp.

Lins, M. P. E., \& Meza, L. A. (2000). Análise Envoltória de Dados e perspectiva de integração no ambiente de apoio à decisão. Rio de Janeiro: COPPE/UFRJ.

Macedo, M. A. S. (2009). Análise do nível de sustentabilidade: um estudo apoiado em Análise Envoltória de Dados (DEA). In: SIMPÓSIO DE ADMINISTRAÇÃO DA PRODUÇÃO, 
LOGÍSTICA E OPERAÇÕES INTERNACIONAIS, 12, 2009, São Paulo. Anais do XII SIMPOI. São Paulo: FGV/EAESP. CD-ROOM.

Macedo, M. A. S., \& Cípola, F. C. (2009). Análise do Desempenho Socioambiental no Setor Siderúrgico Brasileiro. Revista de Contabilidade e Organizações (RCO), 3(7), 58-75.

Macedo, M. A. S., Cípola, F. C., \& Ferreira, A. F. R. (2008). Análise do Desempenho Organizacional sob as Perspectivas Sócio-Ambiental e de Imagem Corporativa: um estudo apoiado em DEA sobre os seis maiores bancos no Brasil. REGE - Revista de Gestão USP, 15, 2008.

Meza, L. A., Biondi, L., Neto, Soares de Mello, J. C. C. B., Gomes, E. G., \& Coelho, P. H. G. (2003). SIAD - Sistema Integrado de Apoio à Decisão: uma implementação computacional de modelos de análise de envoltória de dados. In: SIMPÓSIO DE PESQUISA OPERACIONAL DA MARINHA, 6, 2003, Rio de Janeiro. Anais do VI SPOLM. Rio de Janeiro: CASNAV. CD-ROOM.

Monzoni, M., Biderman, R., \& Brito, R. (2006). Finanças Sustentáveis e o Caso do Índice de Sustentabilidade Empresarial da Bovespa. In: SIMPÓSIO DE ADMINISTRAÇÃO DA PRODUÇÃO, LOGÍSTICA E OPERAÇÕES INTERNACIONAIS, 9, 2006, São Paulo. Anais do IX SIMPOI. São Paulo: FGV/EAESP. CD-ROOM.

Nascimento, L. F. (2008). O insustentável sustentável. In: ENCONTRO DA ASSOCIAÇÃO NACIONAL DE PÓS-GRADUAÇÃO E PESQUISA EM ADMINISTRAÇÃO, 32, 2008, Rio de Janeiro. Anais do XXXII EnANPAD. Rio de Janeiro: ANPAD. CD-ROOM.

Rodrigues, F. F., Santos, R. B., \& Mello, M. C. B. (2009). Análise do disclosure ambiental da empresa Petrobras no período de 1997 a 2007. In: CONGRESSO USP DE CONTROLADORIA E CONTABILIDADE, 9, 2009, São Paulo. Anais do IX Congresso USP. São Paulo: USP/EAC. CDROOM.

Ruthes, S., \& Nascimento, D. E. (2006). Desenvolvimento Sustentável e os Arranjos Produtivos Locais. In: SIMPÓSIO DE ADMINISTRAÇÃO DA PRODUÇÃO, LOGÍSTICA E OPERAÇÕES INTERNACIONAIS, 9, 2006, São Paulo. Anais do IX SIMPOI. São Paulo: FGV/EAESP. CDROOM.

Sachs, I. (2007). Rumo à Ecossocioeconomia: teoria e prática do desenvolvimento. São Paulo: Cortez.

Santos, A., \& Casa Nova, S. P. C. (2005). Proposta de um Modelo Estruturado de Análise de Demonstrações Contábeis. RAE-eletrônica, 4(1), art. 8. Recuperado em 13 janeiro, 2008, de http://www.rae.com.br/eletronica.

Savitz, A. W. (2007). A empresa sustentável: o verdadeiro sucesso é lucro com responsabilidade social e ambiental. Rio de Janeiro: Elsevier.

Soares de Mello, J. C. C. B., Ângulo-Meza, L., Gomes, E. G., \& Biondi, L., Neto. (2005). Curso de Análise Envoltória de Dados. In: SIMPÓSIO BRASILEIRO DE PESQUISA OPERACIONAL, 37, 2005, Gramado. Anais do XXXVII SBPO. Gramado/RS: SOBRAPO. CD-ROM.

Souza, E. G., Andrade, E. O., \& Cândido, G. A. (2008). As Influências dos Indicadores de Sustentabilidade nas Políticas e Ações para a Geração do Desenvolvimento Local Sustentável: um estudo exploratório nos municípios produtores de leite no estado da Paraíba. In: SIMPÓSIO DE 
ADMINISTRAÇÃO DA PRODUÇÃO, LOGÍSTICA E OPERAÇÕES INTERNACIONAIS, 11, 2008. Anais do XI SIMPOI. São Paulo: FGV/EAESP. CD-ROOM.

Souza, V. S. M., \& Harb, A. G. (2009). Análises multivariadas para estabelecer as correlações entre a saúde pública e a educação ambiental no município de Manaus. In: SIMPÓSIO DE ADMINISTRAÇÃO DA PRODUÇÃO, LOGÍSTICA E OPERAÇÕES INTERNACIONAIS, 12, 2009, São Paulo. Anais do XII SIMPOI. São Paulo: FGV/EAESP. CD-ROOM.

Vellani, C. L., \& Ribeiro, M. S. (2006). A Sustentabilidade e a Contabilidade. In: SIMPÓSIO DE ADMINISTRAÇÃO DA PRODUÇÃO, LOGÍSTICA E OPERAÇÕES INTERNACIONAIS, 9, 2006, São Paulo. Anais do IX SIMPOI. São Paulo: FGV/EAESP. CD-ROOM.

Vergara, S. C. (2009). Projetos e Relatórios de Pesquisa em Administração. 10. ed. São Paulo: Atlas.

WCED. (1987). Our Common Future. Oxford and New York: Oxford University Press.

Data do recebimento do artigo: 29/11/2010

Data do aceite de publicação: 28/11/2011

Revista de Gestão Social e Ambiental - RGSA, São Paulo, v. 5, n. 3, p. 73-89, set./dez. 2011. 